\title{
Technè
}

La science au service de l'histoire de l'art et de la préservation des biens culturels

$43 \mid 2016$

Une Europe de la recherche en sciences du patrimoine

\section{D’AGLAE à New AGLAE}

From AGLAE to new AGLAE

Claire Pacheco, Quentin Lemasson, Brice Moignard, Laurent Pichon, Marie Radepont et Didier Gourier

\section{(2) OpenEdition \\ Journals}

Édition électronique

URL : http://journals.openedition.org/techne/689

DOI : $10.4000 /$ techne.689

ISSN : 2534-5168

Éditeur

C2RMF

Édition imprimée

Date de publication : 1 août 2016

Pagination : 63-69

ISBN : 978-2-7118-6338-9

ISSN : $1254-7867$

\section{Référence électronique}

Claire Pacheco, Quentin Lemasson, Brice Moignard, Laurent Pichon, Marie Radepont et Didier Gourier, "D'AGLAE à New AGLAE », Technè [En ligne], 43 | 2016, mis en ligne le 19 décembre 2019, consulté le 23 juillet 2020. URL : http://journals.openedition.org/techne/689 ; DOI : https://doi.org/10.4000/ techne.689

\section{(c) (i) (9)}

La revue Technè. La science au service de l'histoire de l'art et de la préservation des biens culturels est mise à disposition selon les termes de la Licence Creative Commons Attribution - Pas d'Utilisation Commerciale - Pas de Modification 4.0 International. 
Claire Pacheco

Quentin Lemasson

Brice Moignard

Laurent Pichon

Marie Radepont

Didier Gourier

\section{D'AGLAE à New AGLAE}

From AGLAE to new AGLAE

Résumé. Le développement de l'instrument et des méthodes d'analyse par faisceau d'ions appliquées aux problématiques patrimoniales est au cour de l'activité de l'équipe AGLAE. Le projet New AGLAE a pour objectif de mettre à disposition des chercheurs une ligne de faisceau automatisée et en fonctionnement jour et nuit, ainsi qu'un nouveau système de détection PIXE, PIGE, RBS et IBIL capable d'effectuer de l'imagerie chimique systématique sur plusieurs centimètres carrés.

Mots-clés. New AGLAE, multi-détecteur, imagerie chimique systématique.

\begin{abstract}
The development of the instrument and the methodologies of ion beam analysis applied to Cultural Heritage issues is a major interest of the AGLAE team. The New AGLAE project aims at providing an automated beamline available night and day as well as a new PIXE, PIGE, RBS and IBIL detection system enabling systematic chemical imaging on several $\mathrm{cm}^{2}$.

Keywords. New AGLAE, multi-detector, systematic chemical imaging.
\end{abstract}

\section{Automatisation de la ligne de faisceau}

du Louvre, l'Accélérateur Grand Louvre d'Analyse Élémentaire (AGLAE) a fait l'objet de développements instrumentaux et méthodologiques continus afin d'en améliorer les performances pour l'étude d'objets du patrimoine aux problématiques, spécificités et contraintes particulières (voir article de J.-C. Dran dans ce volume). Lauréat d'un appel d'offre Equipex, le projet New AGLAE s'inscrit pleinement dans cette dynamique. En effet, les demandes d'accès au faisceau d'AGLAE se font de plus en plus nombreuses, ce qui a motivé le premier volet du projet, à savoir l'automatisation de la ligne afin de pouvoir travailler également la nuit. De plus, si la plupart des matériaux essentiellement inorganiques (métaux, pierres, céramiques, verres...) peuvent être étudiés directement sans aucune préparation et ce de façon non-invasive, certains matériaux fragiles comme, typiquement, les couches picturales composées d'un pigment inorganique et d'un liant organique, peuvent être modifiées de façon irréversible par le faisceau incident. C'est la raison pour laquelle la conception et le développement d'un multi-détecteur capable de générer une image chimique systématique font l'objet du second axe du projet New AGLAE.
L'automatisation de la ligne de faisceau permet d'augmenter le temps de faisceau disponible et de gagner en efficacité en termes de réglage quotidien. À l'automatisation s'ajoute donc, en pré-requis, la stabilisation du faisceau. Cette double tâche a fait l'objet d'un appel d'offres remporté par la société Thalès. Les travaux sont prévus en 2017 et impliqueront l'inaccessibilité d'AGLAE aux équipes de recherche pendant plusieurs mois.

À terme, ces travaux imposants rendront possible aux utilisateurs l'accès à AGLAE 24h/24, et ce, 5 jours sur 7. La journée restera consacrée aux ouvres et la nuit permettra l'analyse automatisée de séries d'échantillons ou des cartographies nécessitant des acquisitions longues.

\section{Développement d'un multi-détecteur}

\section{PIXE (Particle Induced X-ray Emission)}

L'une des préoccupations majeures est de générer le moins de modifications du matériau sous l'incidence des particules. Si certains matériaux sont bien connus pour n'avoir aucune

Claire Pacheco, ingénieur de recherche, chef du groupe AGLAE+, département Recherche, C2RMF, FR 3506 New AGLAE CNRS/Ministère de la Culture et de la Communication ; responsable de FIXLAB dans le cadre du projet CHARISMA de 2012 à 2014 (claire.pacheco@culture.gouv.fr). Quentin Lemasson (quentin.lemasson@culture.gouv.fr), Brice Moignard (brice.moignard@culture.gouv.fr) et Laurent Pichon (laurent. pichon@culture.gouv.fr), ingénieurs d'étude, AGLAE, département Recherche, C2RMF, FR 3506 New AGLAE CNRS/Ministère de la Culture et de la Communication. Marie Radepont, post-doctorante, C2RMF et CRCC (projet de recherche CORDOBA - Labex Patrima) (marie. radepont@culture.gouv.fr). Didier Gourier, professeur à l'École de Chimie ParisTech, directeur de la fédération de recherche FR 3506 New AGLAE CNRS/Ministère de la Culture et de la Communication (didier.gourier@chimie-paristech.fr). 
réaction visible pendant l'analyse (céramiques, pierres, métaux...), d'autres, comme les verres, peuvent parfois être marqués localement, le phénomène s'avérant souvent réversible à la lumière. D'autres matériaux, comme le blanc de plomb, sont notoirement instables sous le faisceau ${ }^{1}$. Dans le but de diminuer le nombre de particules incidentes susceptibles de modifier le matériau cible, un multi-détecteur, représenté en figure 1, a été conçu et réalisé par l'équipe AGLAE². La possibilité d'additionner les spectres des quatre détecteurs de haute énergie peut présenter plusieurs intérêts pour l'utilisateur. En effet, dans le cas d'étude d'un matériau fragile, une information peut être enregistrée à un niveau exploitable avant que le matériau ne soit modifié de façon irréversible. Si le matériau est peu sensible au faisceau, la multiplication du nombre de détecteurs peut permettre d'augmenter la statistique et les limites de détection. En corollaire, le temps d'analyse peut être diminué pour atteindre le même niveau d'information obtenu avec l'ancien système de détection. Le nombre d'échantillons étudiés en une journée peut donc être augmenté, ce qui est un réel bénéfice pour les études portant sur des séries d'échantillons nécessitant un nombre de points suffisamment conséquent pour pouvoir réaliser des analyses statistiques représentatives comme les études de provenance.

Avec les filtres ad hoc, les détecteurs de haute énergie peuvent être utilisés indépendamment ou également regroupés par 2, 3 ou 4, permettant de mettre en exergue certains éléments dont les limites de détection ne sont pas suffisantes pour un seul détecteur. Par exemple, dans un alliage à base de cuivre, un filtre de $200 \mu \mathrm{m}$ d'aluminium sur un détecteur combiné à un filtre de $25 \mu \mathrm{m}$ de cobalt sur un autre permettent d'obtenir d'une part la composition élémentaire de la matrice et d'autre part les éléments traces lourds. Les éléments légers sont détectés au moyen du détecteur de basse énergie sous flux d'hélium.

L'utilisation du PIXE pour effectuer une recherche de provenance est l'une des applications récurrentes de l'analyse par faisceau d'ions à AGLAE. Dans le cadre de CHARISMA, la campagne de mesures CALLAÏS, souscrite par S. Dominguez Bella de l'université de Cadix en Espagne, avait pour objectif d'identifier la source géologique d'objets néolithiques en variscite issus de sites archéologiques datant du Ve au III ${ }^{\mathrm{e}}$ millénaire avant notre ère dans les régions de Barcelone, Huelva et Zamora. Des matériaux géologiques de référence ont également été analysés afin d'enrichir la base de données existante. Cette campagne, qui s'intégrait dans un projet de recherche plus vaste, a permis de démontrer que les objets n'avaient pas été façonnés à partir de matière première issue des gisements géographiquement les plus proches, certains étant distants de plusieurs centaines de kilomètres ${ }^{3}$.

\section{RBS (Rutherford Backscattering Spectroscopy)}

Le système actuel permet d'utiliser ensemble ou séparément deux détecteurs RBS. Le premier est un détecteur annulaire situé dans la ligne de faisceau à un angle de diffusion égal à $170^{\circ}$, tel qu'il a été conçu antérieurement pour l'étude des patines volontaires des alliages à base de cuivre de l'époque romaine ${ }^{4}$. Si nécessaire, un détecteur RBS annexe peut se substituer à un détecteur PIXE à un angle de diffusion de $130^{\circ}$, permettant de s'affranchir du signal de la fenêtre d'extraction (pour mesurer l'azote par exemple).

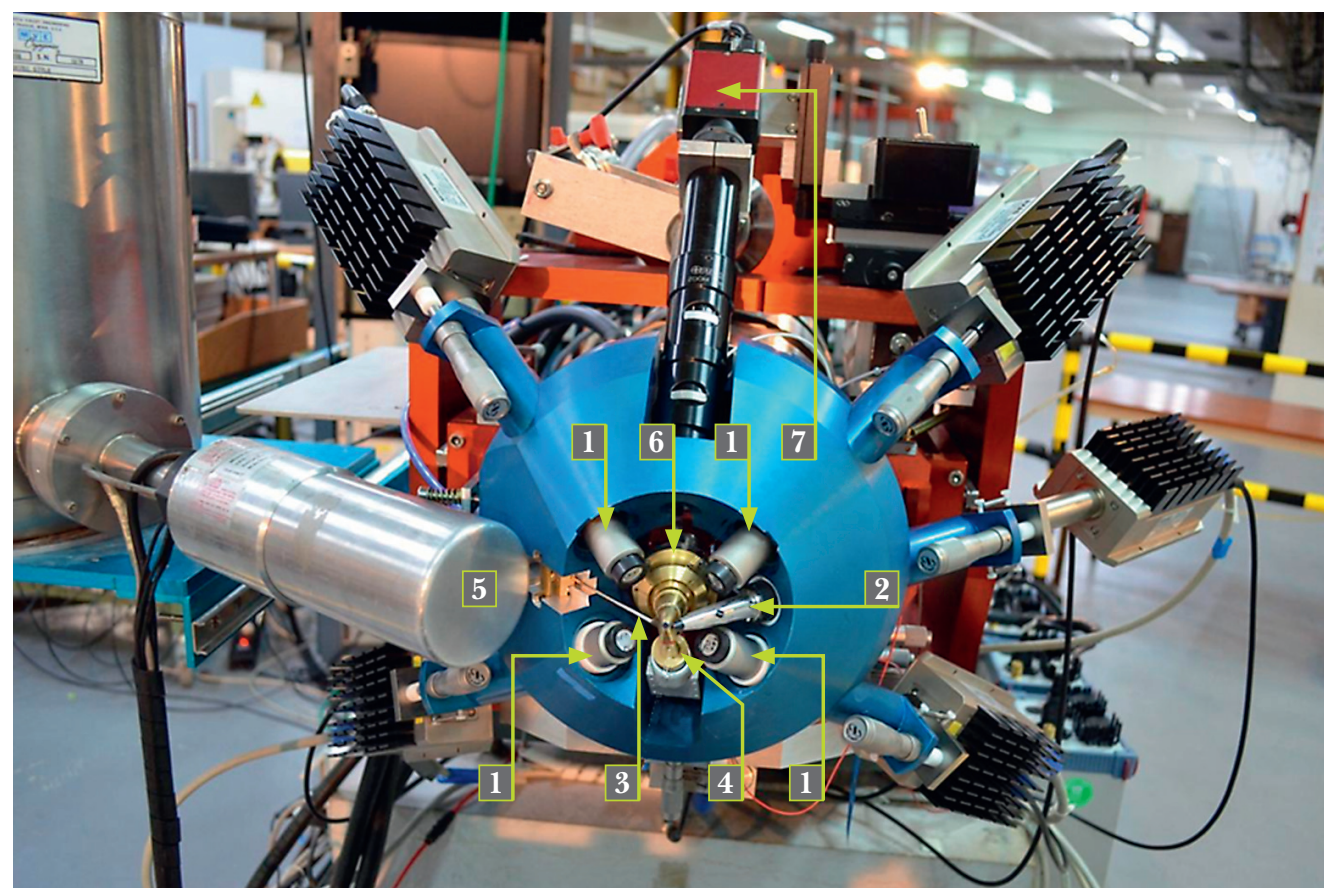

Fig. 1. Extrémité de la ligne de faisceau extrait d'AGLAE arborant le nouveau multidétecteur composé de 1 : quatre détecteurs PIXE de haute énergie ;

2 : un détecteur PIXE de basse énergie ; 3: une fibre optique permettant la collecte du signal d'IBIL;

4: un détecteur de dose ; 5 : un détecteur gamma ; 6 : un détecteur RBS annulaire, situé dans la ligne et invisible sur la photo ;

7 : une caméra permettant de visualiser la zone d'analyse avec précision. 


\section{IBIL (Ion Beam Induced Luminescence)}

L'un des effets de l'interaction particules-matière peut être l'émission d'un rayonnement électromagnétique dans le domaine du visible. On parle alors d'ionoluminescence ou IBIL (Ion Beam Induced Luminescence) ${ }^{5}$. Ce phénomène, sensible à l'environnement chimique des atomes concernés, peut apporter, combiné au PIXE, des informations sur la spéciation (degré d'oxydation, environnement) de certains éléments ${ }^{6}$. En effet, des phases spécifiques peuvent être identifiées grâce à cette technique. Par exemple, le rutile et l'anatase présentent le même spectre PIXE mais des spectres d'IBIL bien caractéristiques. Des ultra-traces, indétectables au PIXE car leurs limites de détection sont inférieures au ppm, peuvent également être mises en évidence par IBIL et s'avérer révélatrices d'une provenance (par exemple, le manganèse dans la calcite ou l'apatite, ou bien les terres rares dans les zircons). Enfin, les modifications de matériau induites par le faisceau d'ions peuvent être mises en évidence par cette méthode. En effet, l'intensité du signal d'ionoluminescence diminue avec le temps d'analyse et la forme du spectre évolue en fonction des défauts créés par l'interaction entre les ions et la matrice ${ }^{7}$.

Sur AGLAE, un système de détection d'IBIL a été intégré au multi-détecteur ${ }^{8}$. Il est constitué d'une fibre optique qui collecte le signal à un angle de $45^{\circ}$ par rapport à l'axe du faisceau. Celle-ci est choisie en fonction du domaine spectral d'intérêt (UV-visible ou visible-proche IR) et transporte le signal jusqu'au spectromètre Ocean Optics QE 65000. Les spectres sont enregistrés pour chaque point analysé simultanément avec les spectres PIXE correspondants. L'une des premières applications de l'IBIL à AGLAE a été l'étude du lapis-lazuli du trésor égyptien de Tôd ${ }^{9}$. La combinaison PIXEIBIL a permis non seulement d'identifier la provenance du lapis-lazuli en Afghanistan, mais également de déterminer que les produits d'altération sont principalement issus de l'oxydation de la pyrite $\mathrm{FeS}_{2}$.

\section{Imagerie systématique}

Jusqu'à présent, les analyses sur AGLAE s'effectuaient point par point. Plusieurs points d'analyse étaient alors nécessaires pour pouvoir faire une moyenne et obtenir un résultat représentatif. La cartographie était également possible en translatant l'objet en X et Y devant le faisceau focalisé sur une cinquantaine de $\mu \mathrm{m}$ de diamètre ${ }^{10}$.

L'équipe AGLAE a adapté un système d'acquisition multiparamétrique fonctionnant en List Mode, signifiant que pour chaque événement enregistré (photon X ou gamma, particule rétrodiffusée...), la position $\{\mathrm{X}, \mathrm{Y}\}$ du faisceau sur la cible est simultanément enregistrée, rendant ainsi l'imagerie chimique systématique accessible.

\section{Un nouveau système de balayage du faisceau}

Le faisceau est dorénavant défléchi verticalement au moyen de bobines magnétiques situées dans la ligne. À une certaine vitesse, la marque ponctuelle du faisceau n'est plus visible et l'on obtient un « pinceau » vertical d'une dimension maximale de $500 \mu \mathrm{m}$. Afin de couvrir la zone d'intérêt, l'objet est mécaniquement déplacé horizontalement. Au bout de la ligne balayée, la cible effectue un saut mécanique vertical équivalent à la hauteur du pinceau, puis le balayage horizontal reprend en sens inverse. Cette combinaison de balayages magnétique et mécanique permet de couvrir une zone sur toute la longueur de débattement mécanique du chevalet d'AGLAE, à savoir $20 \times 20 \mathrm{~cm}^{2}$ avec une résolution maximale de $6 \times 3 \mu \mathrm{m}^{2}$, ce qui est particulièrement adapté aux objets du patrimoine dont les zones d'intérêt peuvent atteindre quelques centimètres carrés.

Deux types de balayages sont opérationnels. Le premier, rapide, ne permet pas d'effectuer des traitements quantitatifs des cartographies obtenues, la dose n'étant pas identique pour chaque pixel. Ce mode de balayage permet de localiser quasi instantanément des zones qui peuvent faire l'objet strict de l'étude ou, à l'inverse, dont il faut s'affranchir car elles fausseraient les mesures. L'autre mode de balayage, moins rapide, permet de fixer une valeur de dose par colonne, la hauteur de celle-ci étant celle du pinceau. L'objet n'est déplacé mécaniquement qu'au moment où cette valeur est atteinte, en dépit des fluctuations du faisceau inhérentes à la machine elle-même. Ce mode de balayage assure une dose homogène sur tous les pixels, rendant possible le traitement quantitatif de la cartographie.

\section{Extraction et traitement quantitatif des données}

Les données générées sont extraites en format EDF (pour ESRF Data Format), permettant ainsi une manipulation aisée par les outils en accès libre tels que PyMCA ${ }^{11}$ ou les logiciels «maison » de traitement et/ou de visualisation des données, développés par l'équipe AGLAE pour ses besoins spécifiques. Au moment de l'extraction, la résolution peut être diminuée, augmentant ainsi la statistique par pixel.

Le logiciel AGLAEMap (fig. 2) permet de rapidement sélectionner et visualiser des données enregistrées par chaque détecteur sur une même zone (PIXE, PIGE, RBS ou IBIL). Le spectre somme correspondant s'affiche sous cette dernière. L'utilisateur peut sélectionner un pic du spectre, la répartition spatiale de l'élément correspondant se met alors automatiquement à jour. Si seule une zone spécifique fait l'objet de l'étude, l'utilisateur peut sélectionner les pixels de cette zone et enregistrer les données de chaque détecteur pour cette zone. Le traitement de données s'effectue alors comme pour une analyse ponctuelle.

Le schéma en figure 3 illustre les différentes étapes pour l'obtention d'une cartographie quantitative à partir des données brutes. En effet, chaque famille de données (PIXE, PIGE, 


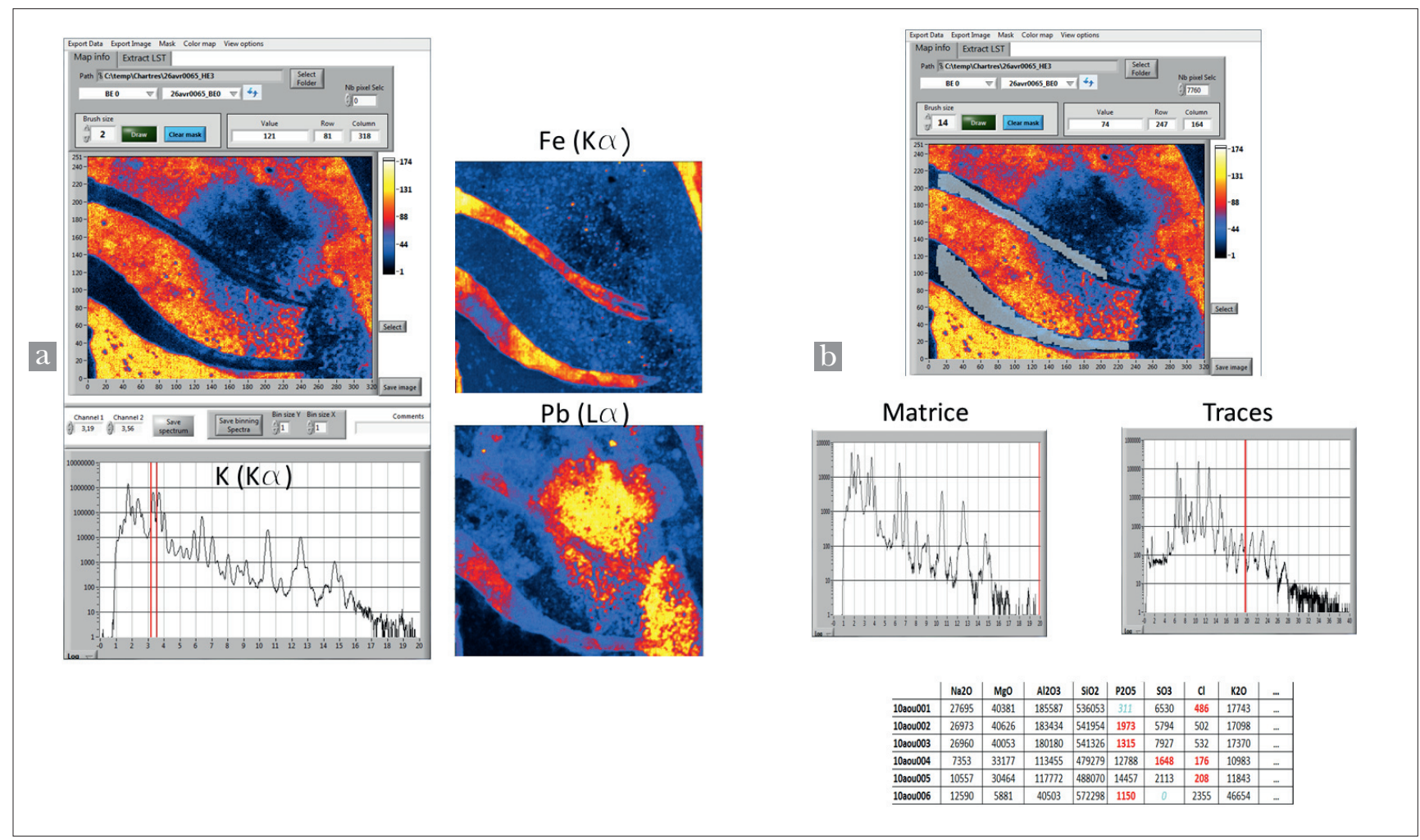

Fig. 2. Exemple de traitement de données réalisables par le logiciel AGLAEMap. a) Sur le spectre PIXE de la zone analysée, un pic est sélectionné. La cartographie se met alors à jour et révèle la répartition spatiale de l'élément choisi. Ici, 3 éléments ont été choisis l'un après l'autre $(\mathrm{K}, \mathrm{Fe}$ et $\mathrm{Pb})$. b) Sur la cartographie, une zone d'intérêt est sélectionnée (en gris sur l'image). Les données enregistrées par chaque détecteur sur cette zone-là uniquement peuvent être traitées comme des analyses ponctuelles.

RBS ou IBIL) doit être traitée par un outil conçu et développé par l'équipe AGLAE qui fait appel à un moteur de calcul spécifique. Les données PIXE sont ainsi traitées par le logiciel maison TRAUPIXE ${ }^{12}$ qui fait appel au moteur de calcul GUPIXWin ${ }^{13}$. L'image quantitative est reconstruite par DataImaging, comme illustré en figures 4 et 5 par deux exemples. Une campagne de mesures a été initiée par J. Van Bennekom du Rijsksmuseum à Amsterdam sur une plaque en argent du XVI ${ }^{\mathrm{e}}$ siècle. Celle-ci est située sous le centre de table connu sous le nom de MerkelscheTafelaufsatz, signé de l'orfèvre Wenzel Jamnitzer. La plaque porte des traces de gravure qui a été volontairement effacée. Aucune technique (photographie en incidence rasante, radiographie, cartographie en XRF) n'a permis de lire le texte. La cartographie PIXE sur AGLAE a donné la possibilité non seulement d'identifier certaines lettres, mais également de valider certaines hypothèses quant au mode de gravure à l'eau-forte et la technique utilisée pour supprimer l'inscription (burin et/ou papier abrasif) ${ }^{14}$. L'imagerie systématique sur AGLAE ouvre de nouveaux champs d'application aux analyses par faisceau d'ions appliquées au patrimoine. Le second exemple est illustré par l'étude d'un vitrail de la cathédrale de Chartres datant du XII ${ }^{\mathrm{e}}$-XIII ${ }^{\mathrm{e}}$ siècle, étude effectuée par C. Loisel du LRMH. Ici, l'une des questions posées concernait la recette du lavis utilisé pour les carnations et les cheveux : s'agissait-il de grisaille diluée ou avait-il été réalisé à partir de silicate de plomb ? L'image permet, d'un coup d'œil, de localiser le

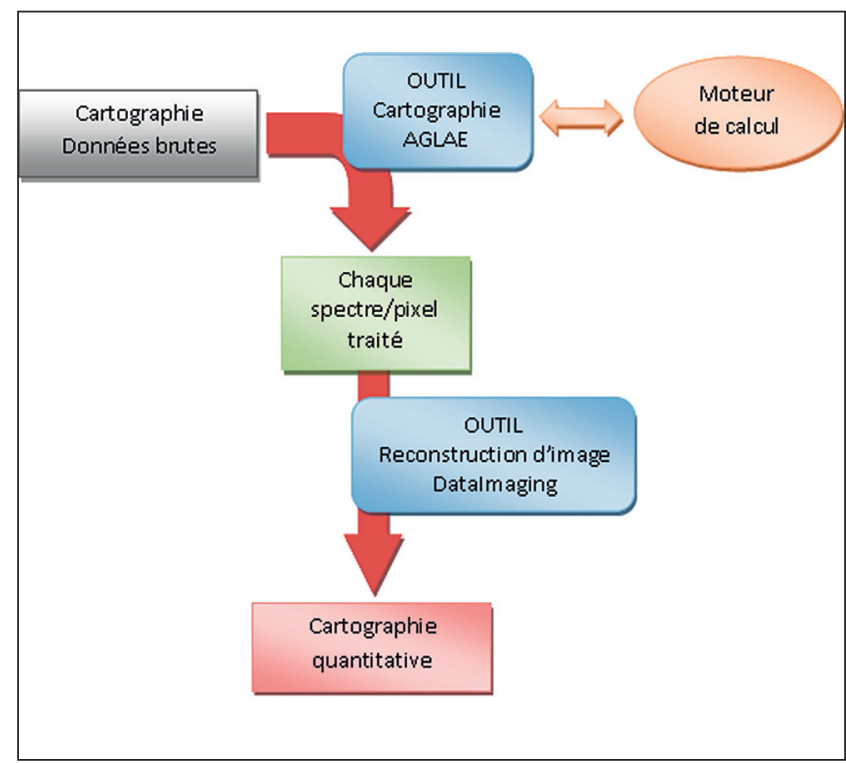

Fig. 3. Étapes et outils nécessaires à l'obtention de cartographies quantitatives à partir de données brutes. Les outils qui font appel aux moteurs de calcul (GUPIXWin pour le PIXE et Data Furnace pour le RBS) et permettant de reconstruire les images à partir des données traitées ont été développés par l'équipe AGLAE. 


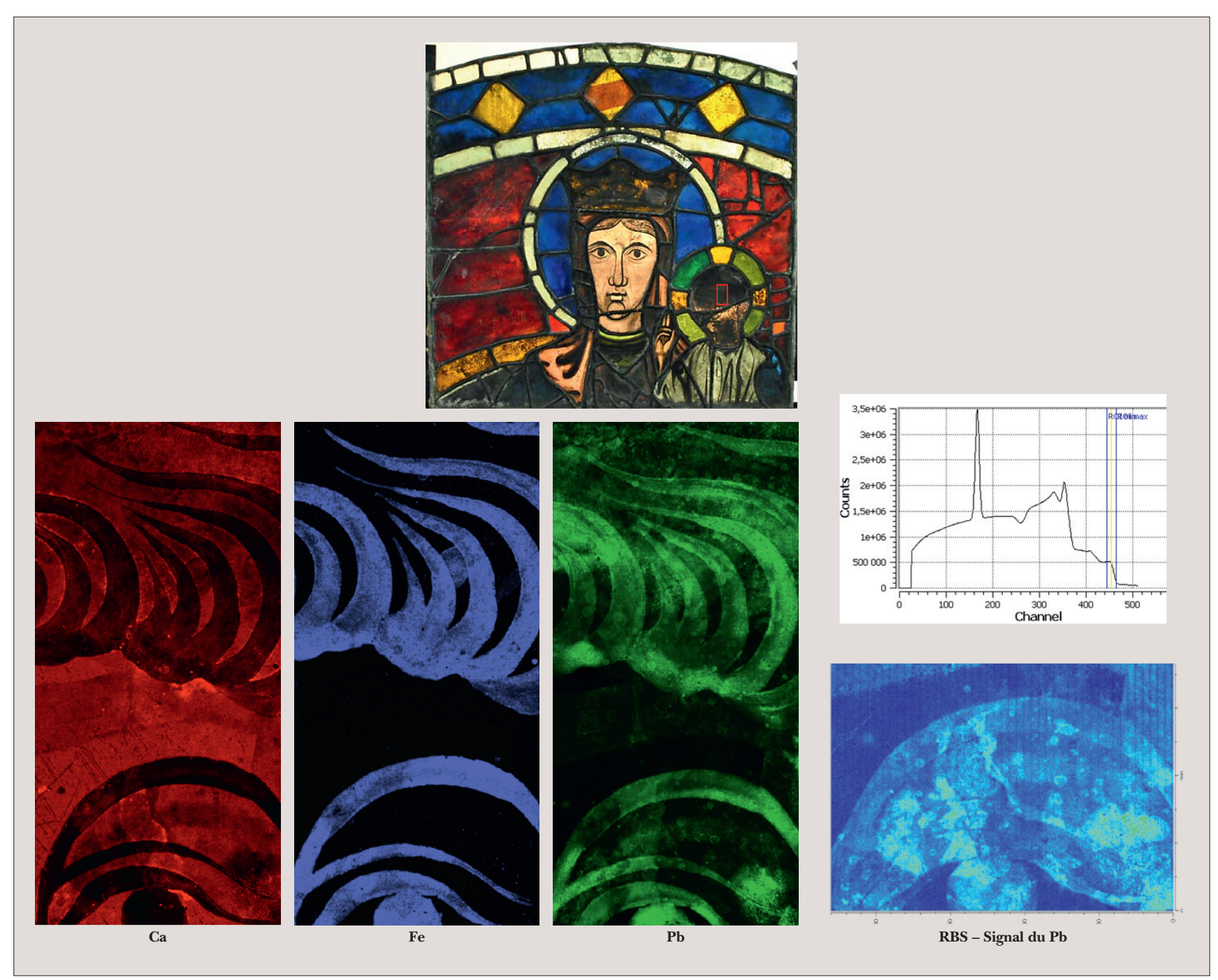

Fig. 4. Cartographie de 6 × $3 \mathrm{~cm}^{2}$ réalisée sur un vitrail de la cathédrale de Chartres du XII ${ }^{\mathrm{e}}$ XIII ${ }^{\mathrm{e}}$ siècles (C C. Loisel - LRMH). Reconstruction de l'image chimique quantitative des éléments $\mathrm{Ca}$, Fe et $\mathrm{Pb}$ par DataImaging après traitement des données par le moteur GUPIXWin. La cartographie RBS révèle la répartition spatiale du plomb en surface et sur quelques dizaines de $\mu \mathrm{m}$ de profondeur.

plomb à la fois dans le lavis et la grisaille, mais dans cette dernière le fer uniquement, validant la seconde hypothèse. L'image obtenue à partir des données RBS brutes représente la répartition spatiale du plomb en surface et à quelques dizaines de micromètres de profondeur. Cette image se superpose à celle du plomb obtenue en PIXE, mais d'autres motifs apparaissent. Pour comprendre et interpréter cette image, chaque spectre contenu dans chaque pixel doit être traité. Pour atteindre cet objectif, l'équipe AGLAE a choisi le moteur de calcul DataFurnace, développé par l'université de Surrey ${ }^{15}$. Afin de mettre au point la méthodologie, un modèle simple a été choisi dans le cadre du programme de recherche CORDOBA (voir l'article de L. Robinet et al. dans ce volume) : une feuille d'argent déposée sur une lame de verre dont une partie a été volontairement repliée. Une cartographie RBS est réalisée avec des particules alpha de $3 \mathrm{MeV}$ et un détecteur à un angle de diffusion de $130^{\circ}$. D'après les caractéristiques données par le fabricant, la feuille d'argent a une épaisseur de $220 \mathrm{~nm}$, ce qui est confirmé par la mesure ponctuelle de l'épaisseur par RBS : $220 \pm 50 \mathrm{~nm}$ pour la feuille simple et $410 \pm 50 \mathrm{~nm}$ pour la double. La reconstruction par DataImaging de l'épaisseur de la feuille d'argent sur la zone analysée montre bien la zone où la feuille est double et les mesures en adéquation avec les valeurs précédentes. Ceci permet de valider la

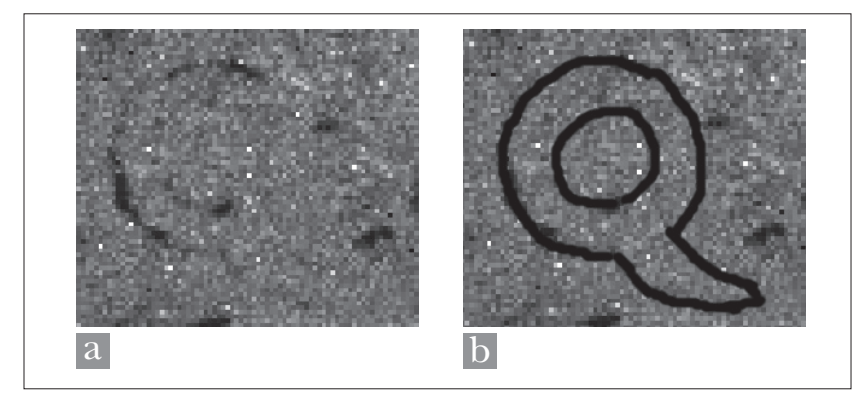

Fig. 5. Cartographie de la raie Ka du cuivre obtenue sur le spectre somme PIXE de haute énergie ; a) le contour d'une lettre se distingue ; b) il s'agit de la lettre $Q$. 
méthodologie et ouvre de réelles perspectives quant à l'imagerie RBS couplée aux autres techniques.

Quant aux spectres IBIL et PIGE, si les premiers peuvent être traités par l'algorithme NNMA (Non-Negative Matrix Approximation $)^{16}$ inclus dans le logiciel PyMCA, la méthodologie et l'outil sont à concevoir et à développer pour le PIGE. L'équipe AGLAE s'attellera à cette tâche dès l'acquisition de nouveaux détecteurs gamma planifiée après l'installation de la nouvelle ligne de faisceau en 2017, réalisée en collaboration avec Thalès.

\section{Conclusion et perspectives}

L'équipe AGLAE conçoit et développe l'instrument et les méthodes d'analyse par faisceau d'ions dans le but d'en repousser les limites actuelles pour les applications patrimoniales. Les projets menés dans le cadre de CHARISMA ont mis en avant la demande de développement de l'IBIL et ont également permis d'effectuer des études originales où l'imagerie était indispensable. Le multi-détecteur est opérationnel et a servi dans de nombreuses études du programme CHARISMA. Un outil de cartographie des données quantitatives RBS est en cours de développement par l'équipe AGLAE et devrait être prochainement opérationnel.

La prochaine grande étape aura lieu en 2017, avec l'installation de la nouvelle ligne de faisceau New AGLAE, plus stable et automatisée, réalisée avec Thalès. Par la suite, de nouveaux détecteurs gamma viendront enrichir le multidétecteur; le traitement des cartographies PIGE, ainsi que le couplage total PIXE-PIGE, seront au centre des préoccupations de l'équipe AGLAE pour 2017-2018, l'objectif final étant de tendre vers le $"$ Total IBA ${ }^{17}$ qui prend en compte les données PIXE et PIGE au cours du traitement des données RBS.

L'installation New AGLAE accueillera de nouveaux utilisateurs français et, dans le cadre du programme IPERION CH (H2020), des chercheurs européens. Ce même programme permet également des activités de recherche conjointes. D'abord, AGLAE est engagée, au côté d'IPANEMA et de BNC-Wigner, dans une tâche consistant à concevoir et développer un porte-objet/échantillon dynamique qui, lors d'une cartographie, positionne la cible de façon à ce que, à chaque point, la zone analysée soit à la bonne distance et perpendiculaire au faisceau. Puis, en collaboration avec ATOMKI-HAS, les modifications des matériaux induites par faisceau d'ions seront un centre d'intérêt majeur qui permettra d'établir les conditions expérimentales optimales pour diminuer les risques, rendant ainsi possible l'étude de matériaux fragiles.

\section{Remerciements}

L'équipe AGLAE souhaite remercier Claudine Loisel du LRMH, Julien Colaux de l'Ion Beam Centre de l'université de Surrey, Dominique Jousse et l'équipe de Thalès. Le projet New AGLAE a été lauréat du programme « Equipex - investissements d'avenir » de l'Agence Nationale de la Recherche (ANR-10-EQPX-22) et a également bénéficié du soutien financier de la Ville de Paris et de Rennes Métropole.

\section{Notes}

1. Beck et al., 2013 ; Calligaro et al., 2015.

2. Pichon et al., 2014.

3. Querré et al., 2014.

4. Mathis et al., 2005 ; ce détecteur a permis, de façon non destructive, d'identifier la patine comme étant de l'oxyde cuivreux $\mathrm{Cu}_{2} \mathrm{O}$ d'une épaisseur de $5,5 \mu \mathrm{m}$.

5. Townsend, 2012.

6. Zucchiatti et al., 2015 ; Quaranta et al., 2008.

7. Joswick et al., 2015.

8. Pichon et al., 2015.

9. Calligaro et al., 2014.

10. Pichon et al, 2010.

11. Solé et al., 2007. 2015.

12. Pichon et al., 2013 ; Pichon et al.,

13. Maxwell et al., 1989.

14. Van Bennekom et al., 2015.

15. Barradas et al., 1998.

16. Lee et al., 1999.

17. Jeynes et al., 2012.

\section{Bibliographie}

Barradas N. P., Marriott P. K., Jeynes C., Webb R.P., 1998, "The RBS data furnace: simulated annealing", Nucl. Instrum. Methods B 136-138, p. 1157-1162.

Beck L., Gutiérrez P. C., Miserque F., Thomé L., 2013, "Proton beam modification of lead white pigments", Nucl. Instr. Methods B 307, p. 20-24.

Calligaro T., MacArthur J. D., Salomon J., 1996, "An improved experimental setup analysis of heavy and light elements beam for the simultaneous PIXE with a $3 \mathrm{MeV}$ proton external beam", Nucl. Instrum. Methods B 109/110, p. 125-128.

Calligaro T., Dran J.-C., Ioannidou E., Moignard B., Pichon L., Salomon J., 2000, "Development of an external beam nuclear microprobe on the Aglae facility of the Louvre museum", Nucl. Instrum. Methods B 161-163, p. 328-333.

Calligaro T., Coquinot Y., Pichon L., PierratBonnefois G., De Campos P., Re A., Angelici D., 2014, "Characterization of the lapis lazuli from the Egyptian treasure of Tôd and its alteration using external $\mu$-PIXE and $\mu$-IBIL", Nucl. Instrum. Methods B 318, p. 139-144.
Calligaro T., Gonzalez V., Pichon L., 2015, "PIXE analysis of historical paintings: Is the gain worth the risk?", Nucl. Instrum. Methods B 363, p. 135-143.

G. Coote, Whitehead N. E., McCallum G. J., 1972, "A rapid method of obsidian characterization by inelastic scattering of protons", J. Radioanal. Chem 12(2), p. 491-496.

G. Deconninck, 1972, "Quantitative analysis by $(\mathrm{p}, \mathrm{X})$ and $(\mathrm{p}, \mathrm{g})$ reactions at low energies", J. Radioanal. Chem. 12(1), p. $157-169$.

Gordon B. M., Kraner H. W., 1972, "On the development of a system for trace element analysis in the environment by charged particle X-Ray fluorescence", J. Radioanal. Chem 12(1), p. 181-188.

Jeynes C., Bailey M. J., Bright N. J., Christopher M. E., Grime G. W., Jones B. N., Palitsin V. V., Webb R. P., 2012, "'Total IBA' - Where are we?",

Nucl. Instrum. Methods B 271, p. 107-118.

Jozwik I., Jagielski J., Gawlik G., Panczer G., Moncoffre N., Ratajczak R., Jozwik P., Wajler A., Sidorowicz A., Thomé L., 2015, "Ion beam-induced luminescence as method of characterization of radiation damage in polycrystalline materials", Nucl. Instrum. Methods B 365, p. $273-277$. 
Katsanos A. A., Xenoulis A., Hadjiantoniou A., Fink R. W., 1976, "An external beam technique for Proton-Induced X-Ray Emission analysis", Nucl. Instrum. Methods 137, p. 119-124.

Lee D. D., Seung H. S., 1999, "Learning the parts of objects by non-negative matrix factorization", Nature 401 (6755), p. 788-791.

Lin E. K., Wang C. W., Teng P. K., Huang Y. M., Chen C. Y., 1992, "Elemental analysis with external-beam PIXE", Nucl. Instrum. Methods B 68, p. 281-284.

Mathis F., Moignard B., Pichon L., Dubreuil O., Salomon J., 2005, "Coupled PIXE and RBS using a $6 \mathrm{MeV} 4 \mathrm{He} 2+$ external beam: a new experimental device for particle detection and dose monitoring", Nucl. Instrum. Methods B 240, p. 532-538.

Maxwell J. A., Campbell J. L., Teesdale W. J., 1989, "The Guelph PIXE software package", Nucl. Instrum. Methods B 43, p. 218-230.

Menu M., Calligaro T., Salomon J., Amsel G., Moulin J., "The dedicated acceleratorbased IBA facility AGLAE at the Louvre”, Nucl. Instrum. Methods B 45 (1990), p. 610-614.
Pichon L., Moignard B., Lemasson Q., Pacheco C., Walter P., 2014,

"Development of a multi-detector and a systematic imaging system on the

AGLAE external beam", Nucl. Instrum. Methods B 318, p. 27-31.

Pichon L., Calligaro T., Gonzalez V., Lemasson Q., Moignard B., Pacheco C., 2015, "Implementation of ionoluminescence in the AGLAE scanning external microprobe", Nucl. Instrum. Methods B 348, p. 68-72.

Pichon L., Calligaro T., Lemasson Q., Moignard B., Pacheco C., 2015, "Programs for visualization, handling and quantification of PIXE maps at the AGLAE facility", Nucl. Instrum. Methods $B$ 363, p. 48-54.

Quaranta A., Dran J.-C., Salomon J., Tonezzer M., Scian C., Beck L., Carturan S., Maggioni G., Della Mea G., 2008, "Ion beam induced luminescence on white inorganic pigments for paintings", Nucl. Instrum. Methods B 266, p. 2301-2305.

Querré G., Calligaro T., Domínguez-Bella S., Cassen S, 2014, "PIXE analyses over a long period: The case of Neolithic variscite jewels from Western Europe (5th-3th millennium BC)", Nucl.
Instrum. Methods B 318, p. 149-156.

Ryan S. R., Fishbeck H. J., Chesnut K., 1985, "Correlation of archaeological ceramics and clays using an external-beam PIXE analysis of the major elemental constituents", Nucl. Instrum. Methods B 10/11, p. 645-649.

Solé V. A., Papillon E., Cotte M., Walter Ph., Susini J., 2007, "A multiplatform code for the analysis of energy-dispersive X-ray fluorescence spectra”, Spectrochim. Acta B 62, p. 63-68.

Townsend P. D., 2012, "Variations on the use of ion beam luminescence", $\mathrm{Nucl}$. Instrum. Methods B 286, p. 35-39.

Van Bennekom J., Lemasson Q., Pichon L., Moignard B., Pappot A., 2014, "The Merkel centerpiece by Wanzel Jamnitzer: proving the existence of a previously unknown inscription using the AGLAE PIXE mapping system", ArtMatters, 6, p. 1-10.

Zucchiatti A., Jiménez-Rey D., Climent-Font A., Martina S., Faietae R., Maggi M., Giuntini L., Calusi S., 2015, "PIXE and IL analysis of an archeologically problematic XIII century ceramic production", Nucl. Instrum. Methods B 363, p. 144-149. 